\title{
FGF21 attenuates high uric acid-induced endoplasmic reticulum stress, inflammation and vascular endothelial cell dysfunction by activating Sirt1
}

\author{
RONG OUYANG ${ }^{1}$, XIAOQIN ZHAO ${ }^{1}$, RONGPING ZHANG $^{1}$, JING YANG $^{2}$, SIYIN LI $^{2}$ and DAIHUA DENG ${ }^{2}$ \\ ${ }^{1}$ Department of Endocrinology, Affiliated Hospital of Nantong University, Nantong, Jiangsu 226001; \\ ${ }^{2}$ Department of Rheumatology, Mianyang Central Hospital, Mianyang, Sichuan 621000, P.R. China
}

Received July 29, 2021; Accepted October 22, 2021

DOI: $10.3892 / \mathrm{mmr} .2021 .12551$

\begin{abstract}
Uric acid (UA) is the final oxidation product of purine metabolism. Hyperuricemia has been previously reported to contribute to vascular endothelial dysfunction and the development of cardiovascular diseases, metabolic syndrome and chronic kidney diseases. In addition, it has been reported that fibroblast growth factor 21 (FGF21) can exert regulatory effects on UA-induced lipid accumulation. Therefore, the present study aimed to investigate the possible role of FGF21 in HUVEC cell injury induced by UA. The study used UA to induce HUVEC cell injury, inhibited sirtuin 1 (Sirt1) expression using EX527 and overexpressed FGF21 by transfection. Subsequently, reverse transcription-quantitative PCR was performed to measure the mRNA expression levels of FGF21, Sirt1 and inflammatory cytokines TNF- $\alpha$, IL- $1 \beta$ and IL-6, whereas western blotting was performed to measure their corresponding protein expression levels including FGF21, Sirt1, NLR family pyrin domain containing 3, pro-caspase1, apoptosis-associated speck-like protein containing a CARD, activating transcription factor 4 , $\mathrm{C} / \mathrm{EBP}$ homologous protein and eukaryotic initiation factor 2. Furthermore, dichloro-dihydro-fluorescein diacetate staining was performed to measure intracellular reactive oxygen species (ROS) generation in HUVECs. The levels of ROS and nitric oxide were also quantified using commercial assay kits. The results demonstrated that overexpression of FGF21 significantly inhibited UA treatment-induced endoplasmic reticulum (ER) stress, inflammation and oxidative stress in HUVECs. Furthermore, overexpression of FGF21 significantly activated Sirt1. The sirt1 inhibitor, EX527, significantly abrogated the suppressive effects of FGF21 overexpression on
\end{abstract}

Correspondence to: Dr Daihua Deng, Department of Rheumatology, Mianyang Central Hospital, 12 Changjia Lane, Mianyang, Sichuan 621000, P.R. China

E-mail: dengdaihua06@163.com

Key words: uric acid, hyperuricemia, fibroblast growth factor 21, sirtuin 1
ER stress, inflammation and oxidative stress in UA-stimulated HUVECs. To conclude, results of the present study suggested that FGF21 may attenuate UA-induced ER stress, inflammation and vascular endothelial cell dysfunction by activating Sirt1. Therefore, FGF21 may be a potential effective target for the future treatment of vascular endothelial cell dysfunction.

\section{Introduction}

Uric acid (UA) is the final oxidation product of purine metabolism (1). It has been reported that high levels of UA in the serum are closely associated with the development of gout and kidney stones (2). UA crystallization triggers robust inflammation and immune activation, which serves an important role in the development of numerous diseases, including hypertension, atherosclerosis and diabetes $(3,4)$. Increasing epidemiological evidence suggests that hyperuricemia is an independent risk factor for the development of cardiovascular diseases, metabolic syndrome and chronic kidney diseases $(5,6)$. Furthermore, hyperuricemia contributes to vascular endothelial dysfunction and the development of hypertension (7). High serum UA levels have been observed in hypertensive adolescents, where UA reduction can be used to treat hypertension (8). Therefore, it is important to determine the role of UA in vascular endothelial cells.

Fibroblast growth factor(FGF) 21 (FGF21)is a hormone-like member of the FGF family that can regulate energy homeostasis, systemic glucose and lipid metabolism $(9,10)$. FGF21 has been reported to alleviate HUVEC apoptosis by inhibiting the Fas signaling pathway, which ameliorates atherosclerosis in apoE-/-mice (11). Furthermore, FGF21 has been found to alleviate oxidized low-density lipoprotein (ox-LDL)-induced HUVEC pyroptosis through the tet methylcytosine dioxygenase (TET2)/ubiquinol cytochrome c reductase core protein I (UQCRC1)/reactive oxygen species (ROS) signaling pathway (12). Another previous study also demonstrated FGF21 to protect HUVECs against high glucose-induced oxidative stress and apoptosis by activating the PI3K/AKT/FOXO3a signaling pathway (13). However, the role of FGF21 in vascular endothelial cell injury induced by UA remains unclear. It has previously been reported that FGF21 knockdown can reverse the inhibitory effects of the microRNA-149-5p inhibitor on 
lipid accumulation induced by high UA levels (14). Therefore, the present study aimed to investigate the role of FGF21 in vascular endothelial cell injury induced by UA.

In the present study, HUVECs were induced using different concentrations of UA to establish a vascular endothelial cell injury model in vitro, which was then applied to investigate the role of FGF21 in vascular endothelial cell injury induced by UA.

\section{Materials and methods}

Cell culture and treatment. The human umbilical vein endothelial HUVEC-C cell line (CRL-1730) was purchased from the American Type Culture Collection and maintained in DMEM (Gibco; Thermo Fisher Scientific, Inc.) supplemented with $10 \%$ fetal bovine serum (Gibco; Thermo Fisher Scientific, Inc.) and $1 \%$ penicillin/streptomycin (Invitrogen; Thermo Fisher Scientific, Inc.). Cells were maintained at $37^{\circ} \mathrm{C}$ in a humidified atmosphere containing $5 \% \mathrm{CO}_{2}$. HUVECs were stimulated with 6,9 and $12 \mathrm{mg} / \mathrm{dl}$ concentrations of UA (cat. no. U2625; Sigma-Aldrich; Merck KGaA) for $24 \mathrm{~h}$ at room temperature. Sirtuin 1 (sirt1) expression was inhibited by EX527 (10 $\mu \mathrm{M}$; Beyotime Institute of Biotechnology) (15). Untreated cells were used as the control group.

Cell transfection. For transfection, the FGF21 overexpression plasmid (Ov-FGF21; $10 \mathrm{nM}$ ) and the control empty vector (Ov- NC; $10 \mathrm{nM}$ ) were purchased from Shanghai GenePharma Co., Ltd. Subsequently, Ov-FGF21 or Ov-NC, were transfected into cells $\left(2 \times 10^{6}\right.$ cells/well $)$ for $48 \mathrm{~h}$ at $37^{\circ} \mathrm{C}$ using Lipofectamine ${ }^{\circledR} 2000$ reagent (Invitrogen; Thermo Fisher Scientific, Inc.) according to the manufacturer's instructions. HUVECs were used for subsequent experimentation $48 \mathrm{~h}$ post-transfection and reverse transcription (RT)-quantitative (q)PCR analysis was performed to confirm transfection efficiency. In addition, cells were co-treated with EX527. After transfection for $48 \mathrm{~h}$, cells were then exposed to UA for $24 \mathrm{~h}$.

$R T-q P C R$. Total RNA was extracted from HUVECs using TRIzol $^{\circledR}$ (Thermo Fisher Scientific, Inc.) following the manufacturer's instructions. RT was performed using the PrimeScript RT reagent kit (Takara Bio, Inc.) according to the manufacturer's instructions. For RT the following temperature protocol was used; $15 \mathrm{~min}$ at $42^{\circ} \mathrm{C}$; followed by $5 \mathrm{~min}$ at $98^{\circ} \mathrm{C}$. The reaction volume was $20 \mu \mathrm{l}$. qPCR was performed using the SYBR $^{\circledR}$ Green Quantitative RT-qPCR Kit (cat. no. QR0100; Sigma-Aldrich; Merck KGaA). The following thermocycling conditions were used for the qPCR: Initial denaturation at $95^{\circ} \mathrm{C}$ for $30 \mathrm{sec}$; followed by 40 cycles at $95^{\circ} \mathrm{C}$ for $5 \mathrm{sec}$ and $60^{\circ} \mathrm{C}$ for $30 \mathrm{sec}$. The reaction volume was $25 \mu \mathrm{l}$. The mRNA expression levels of FGF21, sirt1, tumor necrosis factor $\alpha$ (TNF- $\alpha$ ), interleukin-1 $\beta$ (IL-1 $\beta$ ) and interleukin-6 (IL-6) were quantified using the $2^{-\Delta \Delta \mathrm{Cq}}$ method (16) and normalized to the internal reference gene, GAPDH. Primer sequences were synthesized by Shanghai GenePharma Co., Ltd. and are displayed in Table I.

Western blotting. Total protein was extracted from HUVECs using the RIPA lysis buffer (cat. no. P0013C; Beyotime Institute of Biotechnology) and protein concentration was determined
Table I. Sequences of primers used for reverse transcription-quantitative PCR.

\begin{tabular}{ll}
\hline Gene & \multicolumn{1}{c}{ Sequence $\left(5^{\prime} \rightarrow 3^{\prime}\right)$} \\
\hline FGF21 & F: CTGTGGGTTTCTGTGCTGG \\
& R: CCGGCTTCAAGGCTTTCAG \\
FIRT1 & F: AAGTTGACTGTGAAGCTGTACG \\
& R: TGCTACTGGTCTTACTTTGAGGG \\
TNF- $\alpha$ & F: TCTCGAACCCCGAGTGACAA \\
& R: TATCTCTCAGCTCCACACCCA \\
IL-1 $\beta$ & F: GGCCCTAAACAGATGAAGTG \\
& R: GTAGTGGTGGTCGGAGATTC \\
IL-6 & F: CCTTCTCCACAAGCGCCTTC \\
& R: GGCAAGTCTCCTCATTGAATC \\
GAPDH & F: GGAGCGAGATCCCTCCAAAAT \\
& R: GGCTGTTGTCATACTTCTCATGG
\end{tabular}

FGF21, fibroblast growth factor 21; SIRT1, sirtuin 1; F, forward; $\mathrm{R}$, reverse.

using a BCA Protein Assay Kit (cat. no. P0012A; Beyotime Institute of Biotechnology). Total protein (30 $\mu \mathrm{g} /$ lane) was separated via SDS-PAGE on a $10 \%$ gel (Beyotime Institute of Biotechnology) and transferred onto PVDF membranes (EMD Millipore). The membranes were blocked with 5\% skimmed milk for $2 \mathrm{~h}$ at room temperature, followed by overnight incubation at $4^{\circ} \mathrm{C}$ with primary antibodies. Following incubation with the primary antibody and washing with TBST $(0.1 \%$ Tween-20; $10 \mathrm{~min}$ at room temperature), the membranes were incubated with a goat HRP-conjugated anti-rabbit secondary antibody (1:5,000; cat. no. S0001; Affinity Biosciences) for $2 \mathrm{~h}$ at room temperature. Protein bands were visualized using an ECL Kit (Beyotime Institute of Biotechnology). Protein expression levels were semi-quantified using Image-Pro Plus software version 6.0 (Media Cybernetics, Inc.; Roper Technologies, Inc.). The primary antibodies used were as follows: FGF21 (1:1,000; cat no. ab64857; Abcam), sirt1 (1:2,000; cat. no. ab12193; Abcam), activating transcription factor 4 (ATF4; 1:500; cat. no. ab216839; Abcam), NLR family pyrin domain containing 3 (NLRP3; 1:500; cat. no. ab214185; Abcam), pro-caspase 1 (1:1,000; cat. no. ab179515; Abcam), apoptosis-associated speck-like protein containing a CARD (ASC; 1:1,000; cat. no. ab70627; Abcam), phosphorylated (p)-AKT (1:500; cat. no. ab38449; Abcam), AKT (1:500; cat. no. ab8805; Abcam), p-endothelial nitric oxide synthase (p-eNOS; 1:500; cat. no. ab184154; Abcam), eNOS (1:1,000; cat. no. ab5589; Abcam), GAPDH (1:2,500; cat. no. ab9485; Abcam), C/EBP homologous protein (CHOP; 1:1,000; cat. no. DF6025; Affinity Biosciences), p-eukaryotic initiation factor 2 (p-eIF2A; 1:500; cat. no. AF7188; Affinity Biosciences) and eIF2A (1:1,000; cat. no. AF6087; Affinity Biosciences).

Assessment of ROS and nitric oxide (NO) levels. Intracellular ROS generation was quantified as previously described (17). HUVECs were inoculated in six-well plates $\left(5 \times 10^{5}\right.$ cells/well $)$ and incubated with $10 \mu \mathrm{M}$ dichloro-dihydro-fluorescein diacetate (DCFH-DA; Sigma-Aldrich; Merck KGaA) at $37^{\circ} \mathrm{C}$ 
A

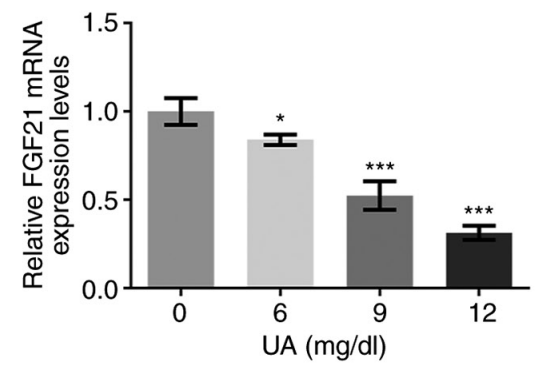

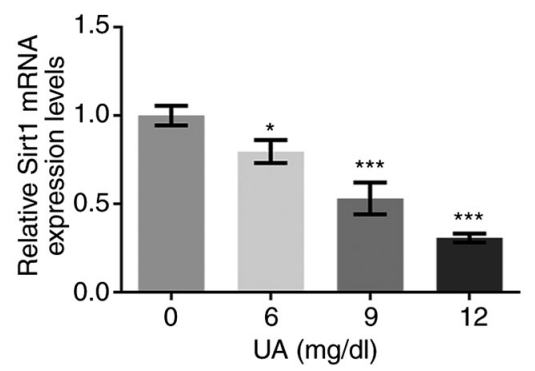
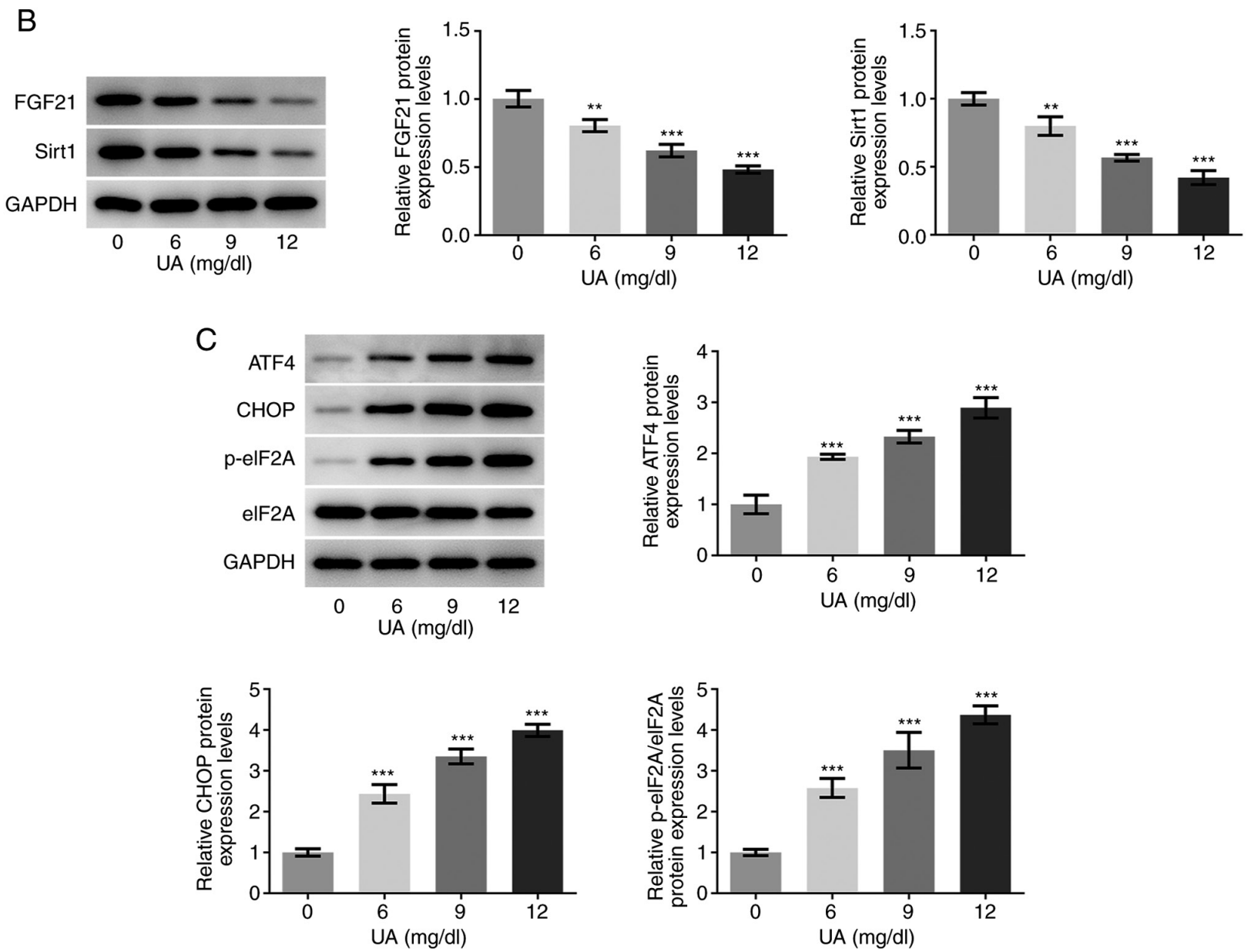

Figure 1. High UA levels inhibits FGF21 and Sirt1 expression levels and promotes endoplasmic reticulum stress in HUVECs. (A) mRNA expression levels of FGF21 and Sirt1 were determined using reverse transcription-quantitative PCR. (B) Protein expression levels of FGF21 and Sirt1 were determined using western blotting. (C) Protein levels of ATF4, CHOP and p-eIF2A/eIF2A were determined using western blotting. ${ }^{*} \mathrm{P}<0.05,{ }^{* *} \mathrm{P}<0.01$ and ${ }^{* * * *} \mathrm{P}<0.001 \mathrm{vs} .0 \mathrm{mg} / \mathrm{dl}$ UA control group. FGF21, fibroblast growth factor 21; UA, uric acid; Sirt1, sirtuin 1; CHOP, eIF2A, eukaryotic initiation factor 2; ATF4, activating transcription factor 4; p-, phosphorylated.

for $30 \mathrm{~min}$ in the dark. In the presence of ROS, DCFH-DA is oxidized and produces fluorescence (18). Cells were washed three times with pre-cooled PBS and ROS levels were determined using a fluorescence microscope (magnification, x200), with excitation and emission wavelengths of 485 and $520 \mathrm{~nm}$, respectively. ROS levels were also quantified using a Fluorometric Intracellular ROS Kit (cat. no. MAK143; Sigma-Aldrich; Merck KGaA), according to the manufacturer's protocols.

NO levels were quantified using a NO Assay Kit (cat. no. A012-1-2; Nanjing Jiancheng Bioengineering Institute.). According to the manufacturer's protocols, the mixed reagents were added sequentially to the samples. After standing at room temperature for $40 \mathrm{~min}$, the samples were centrifuged at $1,000 \mathrm{x} \mathrm{g}$ at $4^{\circ} \mathrm{C}$ for $10 \mathrm{~min}$. The supernatant was removed and color developer was added. After $10 \mathrm{~min}$ at room temperature, the absorbance was detected at $550 \mathrm{~nm}$ using a microplate reader (Bio-Rad Laboratories, Inc.).

Statistical analysis. Statistical analysis was performed using GraphPad Prism 8.0 software (GraphPad Software, Inc.). Data are presented as the mean \pm standard deviation of $\geq$ three independent experiments. One-way ANOVA was used to compare differences between three or more groups followed by Tukey's post hoc test. $\mathrm{P}<0.05$ was considered to indicate a statistically significant difference. 
A

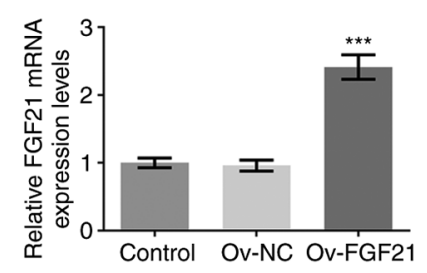

B

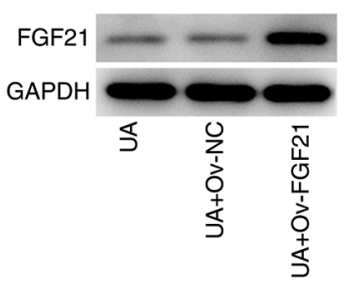

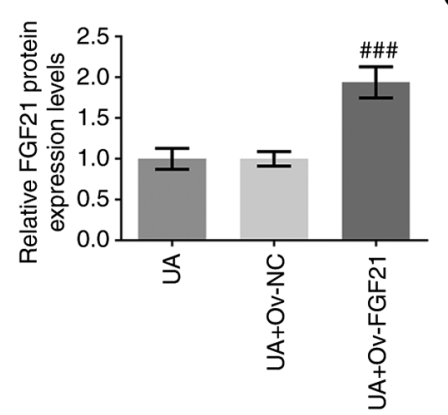

C

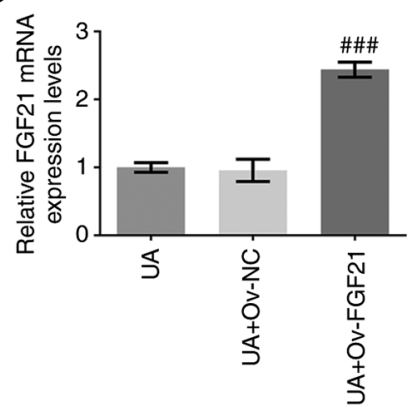

D

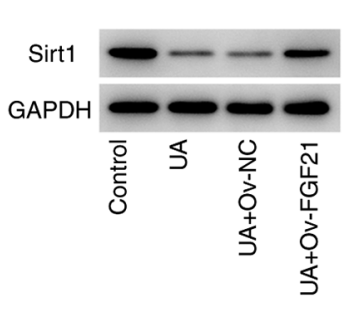

E

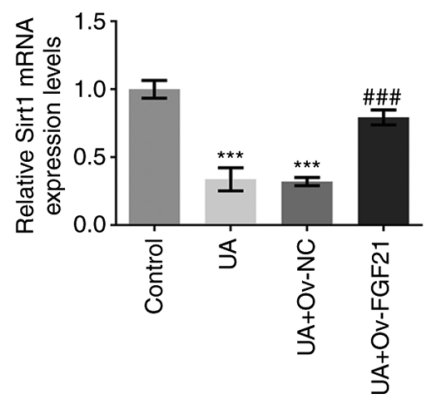

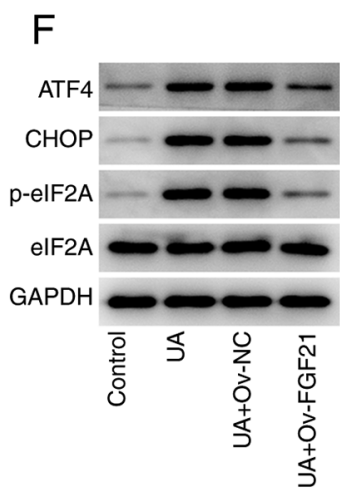
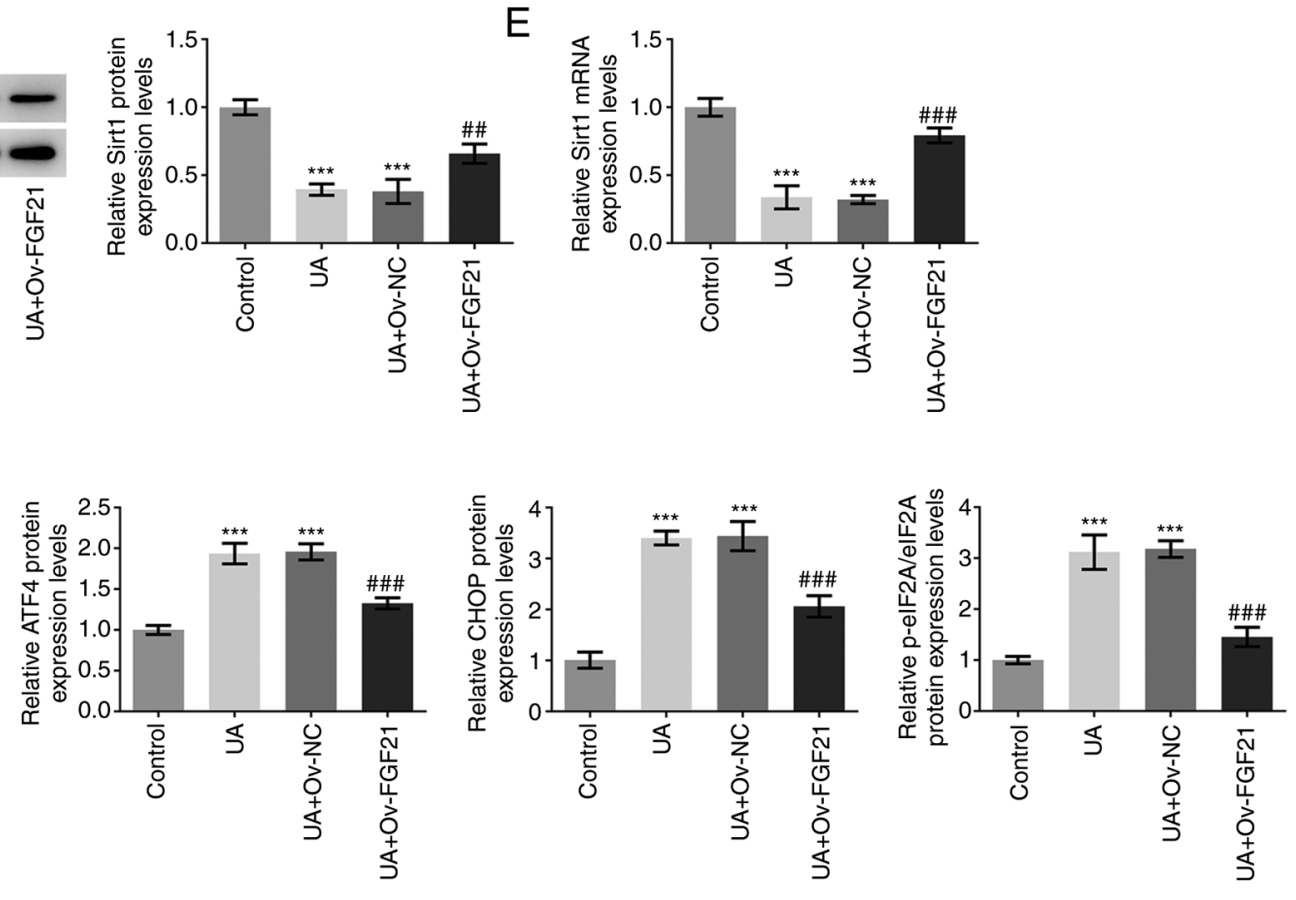

Figure 2. Overexpression of FGF21 induces Sirt1 activation and suppresses endoplasmic reticulum stress in HUVECs. (A) FGF21 mRNA expression levels were determined using RT-qPCR. (B) Western blotting was performed to measure FGF21 protein expression levels. (C) RT-qPCR was performed to detect FGF21 mRNA expression levels. (D) Western blotting was performed to detect Sirt1 protein expression levels. (E) RT-qPCR was performed to detect Sirt1 mRNA expression levels. (F) Western blotting analysis was performed to detect the protein levels of ATF4, CHOP, p-eIF2A and eIF2A.**** $<<0.001$ vs. control; ${ }^{\# \#} \mathrm{P}<0.01$ and ${ }^{\# \# \#} \mathrm{P}<0.001$ vs. UA + Ov-NC. UA, uric acid; FGF21, fibroblast growth factor 21; Sirt1, sirtuin 1; RT-qPCR, reverse transcription-quantitative PCR; ATF4, activating transcription factor 4; eIF2A, eukaryotic initiation factor 2; p-, phosphorylated; NC, negative control; Ov, overexpressed.

\section{Results}

High UA inhibits FGF21 and Sirtl expression levels and promotes endoplasmic reticulum (ER) stress in HUVECs. The mRNA and protein expression levels of FGF21 and sirt1 in UA-stimulated HUVECs were determined via RT-qPCR and western blotting, respectively. As presented in Fig. 1A and B, the mRNA and protein expression levels of FGF21 and sirt1 were significantly decreased following UA stimulation in a dose-dependent manner compared with those in the $0 \mathrm{mg} / \mathrm{dl}$ UA group (control group). Furthermore, the protein expression levels of ER stress-associated proteins ATF4, CHOP and the p-eIF2A/eIF2A ratio were also semi-quantified using western blotting. As shown in Fig. 1C, the protein expression levels of ATF4, CHOP and eIF2A phosphorylation were all significantly increased in UA-stimulated HUVECs, in a dose-dependent manner compared with those in the $0 \mathrm{mg} / \mathrm{dl}$ UA group. Furthermore, when the concentration of UA was $12 \mathrm{mg} / \mathrm{dl}$, the highest difference was observed compared with that in the $0 \mathrm{mg} / \mathrm{dl}$ UA group. Therefore, $12 \mathrm{mg} / \mathrm{dl} \mathrm{UA}$ was selected for subsequent experimentation. These results suggest that the expression levels of FGF21 and sirt1 may be closely associated with the progression of UA-induced vascular endothelial cell injury.

Overexpression of FGF21 activates sirtl and suppresses ER stress in HUVECs. FGF21 was overexpressed via transfection of HUVEC with the Ov-FGF21 plasmid. RT-qPCR analysis demonstrated that the Ov-FGF21 plasmid was successfully transfected into HUVECs, since the Ov-FGF21 plasmid transfection significantly increased FGF21 expression compared with that in HUVECs transfected with Ov-NC (Fig. 2A). FGF21 was significantly upregulated in the UA + Ov-FGF21 group compared with that in the $\mathrm{UA}+\mathrm{Ov}-\mathrm{NC}$ group, indicating that the Ov-FGF21 plasmid was also successfully transfected into UA-induced HUVECs (Fig. 2B and C). Subsequently, Sirtl mRNA and protein expression levels in the transfected cells were measured (Fig. 2D and E). Compared with those 

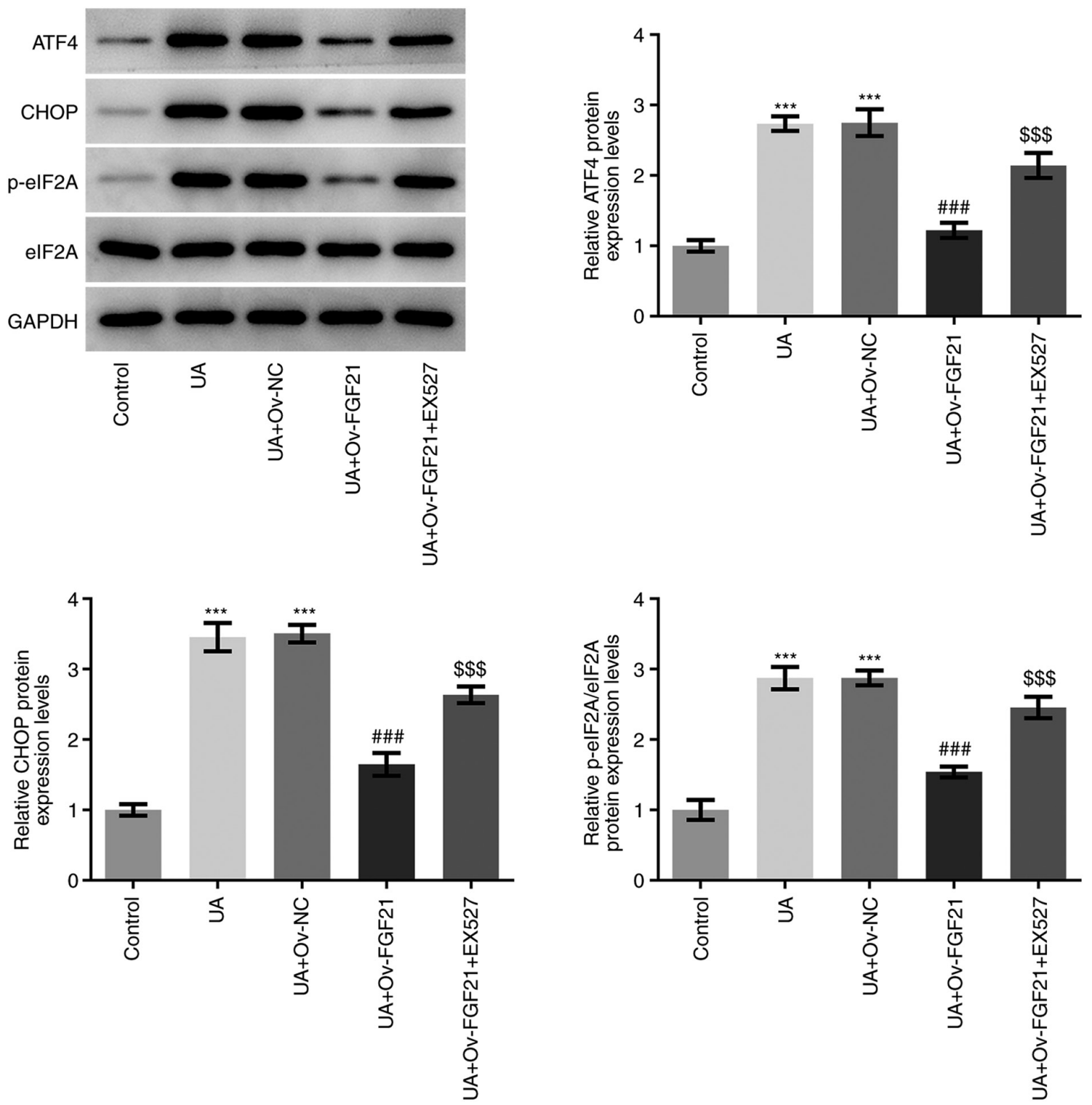

Figure 3. Overexpression of FGF21 attenuates endoplasmic reticulum stress in high UA-induced HUVECs by activating Sirtuin 1. Western blotting was performed to detect the protein levels of ATF4, CHOP, p-eIF2A and eIF2A. ${ }^{* * *} \mathrm{P}<0.001$ vs. control. ${ }^{\# \# \# ~} \mathrm{P}<0.001$ vs. UA $+\mathrm{Ov}-\mathrm{NC}$; ${ }^{\$ \$ \$} \mathrm{P}<0.001 \mathrm{vs}$. UA + Ov-FGF21. FGF21, fibroblast growth factor 21; UA, uric acid; ATF4, activating transcription factor 4; eIF2A, eukaryotic initiation factor 2; Ov, overexpressed; p-, phosphorylated; NC, negative control.

in the UA + Ov-NC group, overexpression of FGF21 was demonstrated to significantly increase sirt1 mRNA and protein expression levels. The protein expression levels of ATF4, CHOP and eIF2A phosphorylation were significantly decreased in the UA + Ov-FGF21 group compared with those in the UA + Ov-NC group (Fig. 2F). Collectively, these results suggest that FGF21 may activate sirt1 and inhibit ER stress.

Overexpression of FGF21 attenuates ER stress and the inflammatory response in high UA-induced HUVECs by activating sirt1. EX527 was used as a sirt1 inhibitor for subsequent experiments. As presented in Fig. 3, the protein expression levels of ATF4, CHOP and eIF2A phosphorylation in HUVECs were significantly higher in the UA + Ov-FGF21 + EX527 group compared with those in the UA + Ov-FGF21 group. These results suggest that EX527 may reverse the suppressive effects of FGF21 overexpression on ER stress in HUVECs.
The mRNA expression levels of TNF $\alpha$, IL-1 $\beta$ and IL- 6 were next detected by RT-qPCR analysis. As presented in Fig. 4A, UA stimulation significantly promoted the expression of inflammatory cytokines TNF $\alpha$, IL-1 $\beta$ and IL-6, compared with that in the control group. Following FGF21 overexpression, the mRNA expression levels of these inflammatory cytokines were significantly suppressed compared with those in the UA + Ov-NC group. However, after EX527 was added, this suppression of inflammatory cytokine mRNA expression levels by FGF21 was significantly reversed compared with that in the UA + Ov-FGF21 group. The protein expression levels of NLRP3, pro-caspase 1 and ASC, which are inflammasome proteins, were measured using western blotting (19). As presented in Fig. 4B, the protein expression levels of NLRP3, pro-caspase 1 and ASC were significantly increased following UA stimulation compared with those in the control group. By contrast, compared with those in the UA + Ov-NC group their 

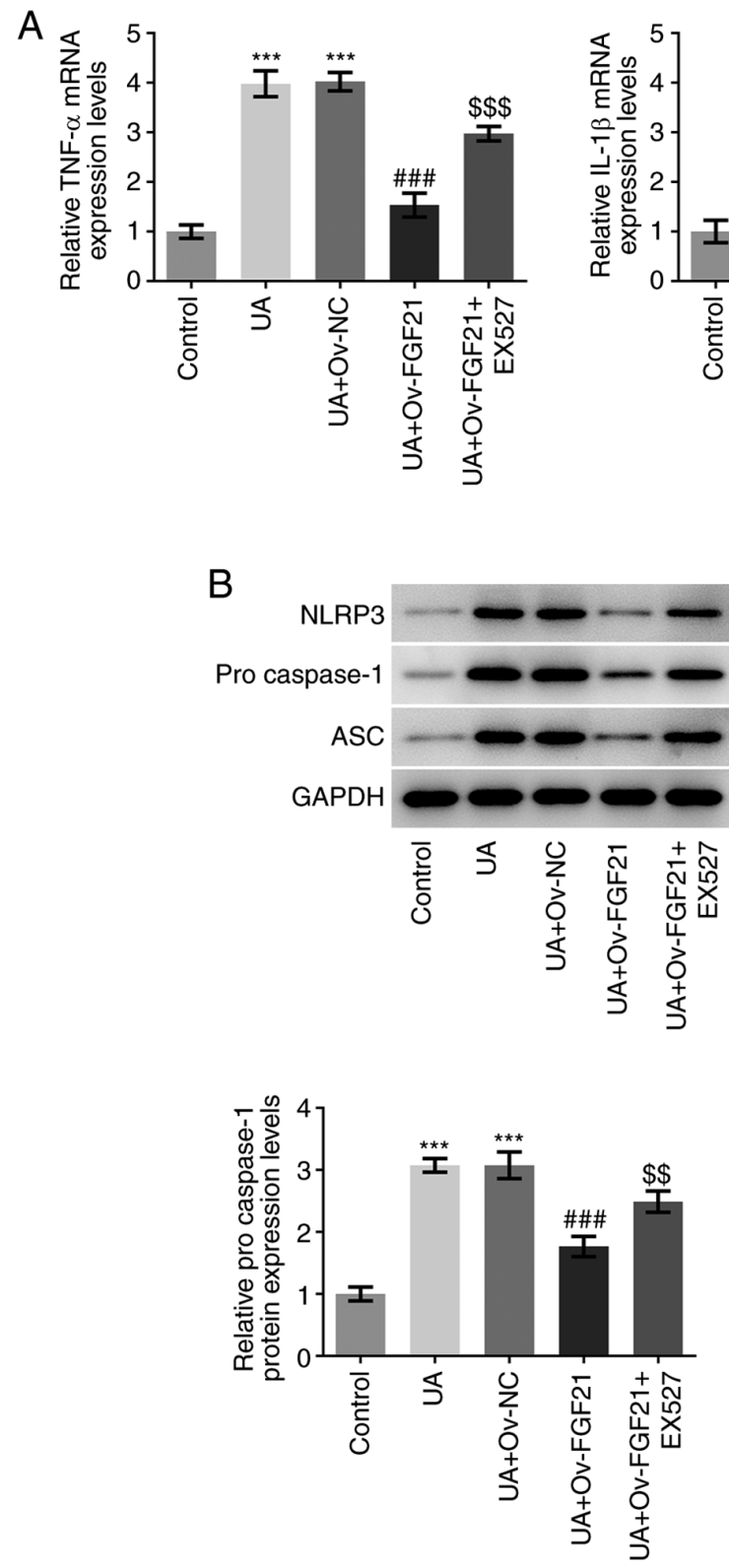
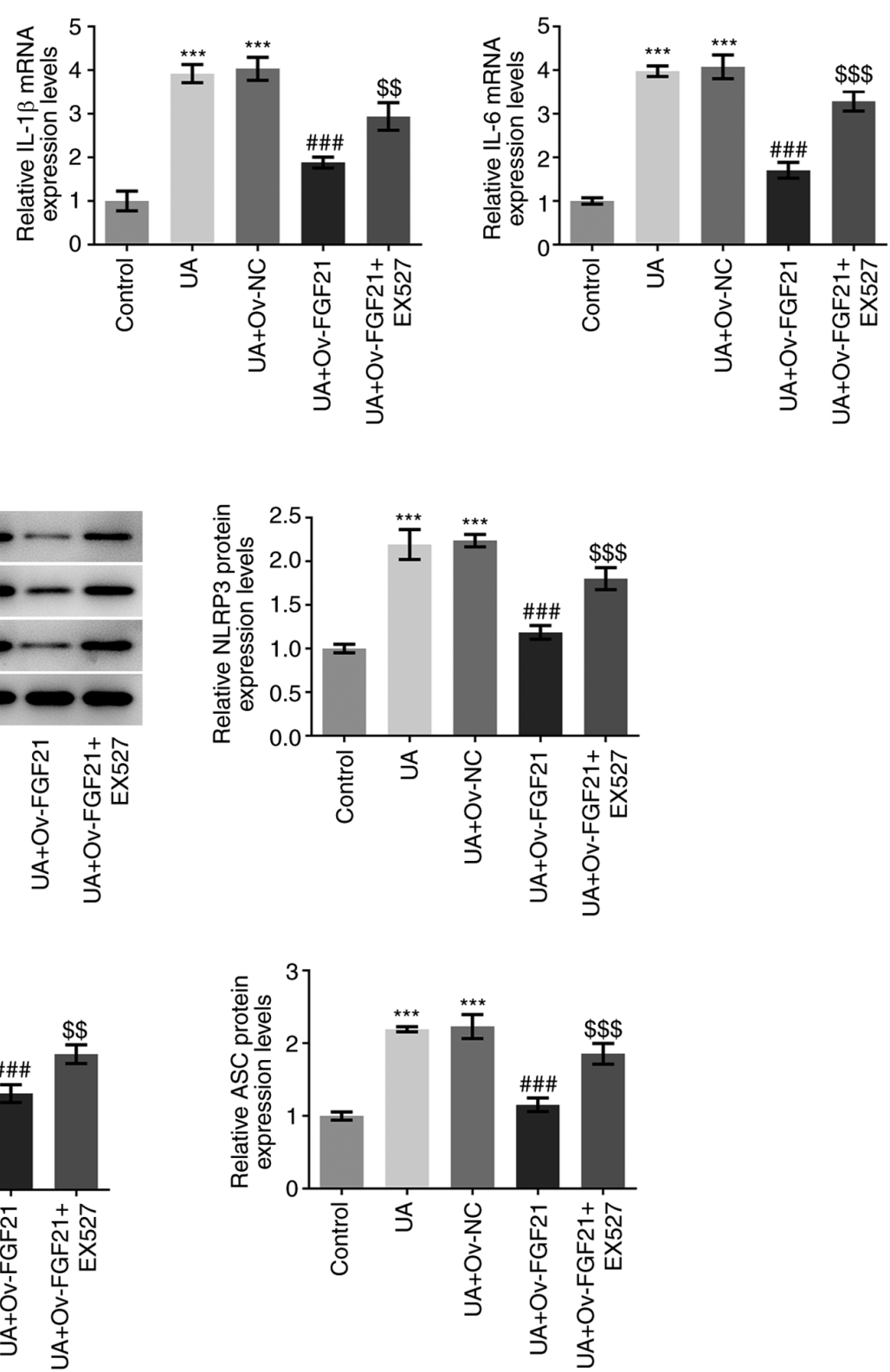

Figure 4. Overexpression of FGF21 attenuates the inflammatory response in high UA-induced HUVECs by activating Sirtuin 1. (A) Reverse transcriptionquantitative PCR analysis was performed to detect the mRNA expression levels of TNF- $\alpha$, IL-1 $\beta$ and IL- 6 . (B) Western blotting was performed to detect the protein expression levels of NLRP3, pro-caspase 1 and ASC. ${ }^{* * *} \mathrm{P}<0.001$ vs. control; ${ }^{\# \#} \mathrm{P}<0.001 \mathrm{vs}$. UA + Ov-NC; ${ }^{\$ \$} \mathrm{P}<0.01$ and ${ }^{\$ \$ S} \mathrm{P}<0.001$ vs. UA + Ov-FGF21. FGF21, fibroblast growth factor 21; UA, uric acid; NLPR3, NLR family pyrin domain containing 3; ASC, apoptosis-associated speck-like protein containing a CARD; Ov, overexpressed; NC, negative control.

protein expression levels were significantly inhibited following the overexpression of FGF21. Furthermore, EX527 significantly reversed the inhibitory effects of FGF21 on the protein expression levels of NLRP3, pro-caspase 1 and ASC compared with those in the UA + Ov-FGF21 group. These results suggest that the overexpression of FGF21 may attenuate ER stress and inflammatory injury in high UA-induced HUVECs by activating Sirt1.

Overexpression of FGF21 attenuates oxidative stress and vascular endothelial cell dysfunction in high UA-induced HUVECs by activating Sirtl. The levels of ROS and NO in HUVECs were measured. Results from DCFH-DA staining and commercial assay kits demonstrated that UA stimulation significantly promoted ROS generation compared with that in the control group. Furthermore, overexpression of FGF21 significantly inhibited ROS generation in UA-stimulated HUVECs compared with that in the UA + Ov-NC group, whereas the sirt1 inhibitor EX527 significantly reversed this effect compared with that in the UA + Ov-FGF21 group (Fig. 5A and B). Collectively, these results suggest that FGF21 can potentially inhibit ROS production by activating Sirt1.

As shown in Fig. 6A, NO levels were significantly decreased following UA stimulation compared with those in the control group. However, NO levels were significantly increased following the overexpression of FGF21 compared with those in the UA + Ov-NC group. Furthermore, treatment with the Sirt1 inhibitor EX527 significantly attenuated NO levels following the overexpression of FGF21 compared with those in the UA + Ov-FGF21 group. In addition, UA stimulation significantly decreased the 
A
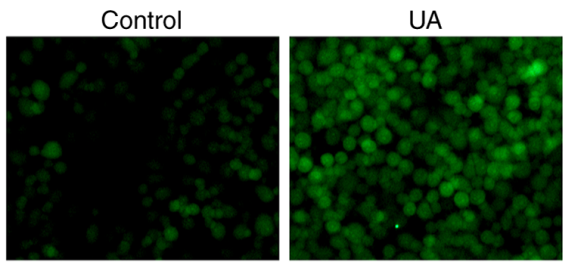

$\mathrm{UA}+\mathrm{Ov}-\mathrm{NC}$

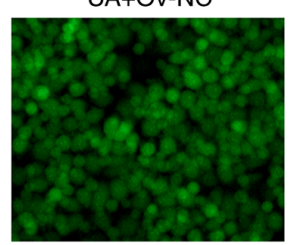

UA+Ov-FGF21

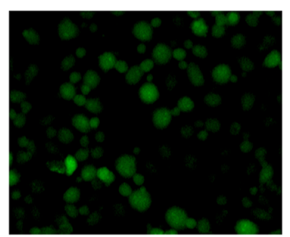

$\mathrm{UA}+\mathrm{Ov}-\mathrm{FGF} 21+\mathrm{EX} 527$

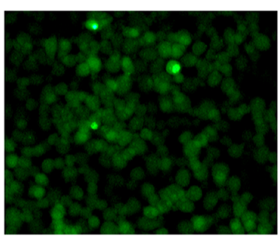

B

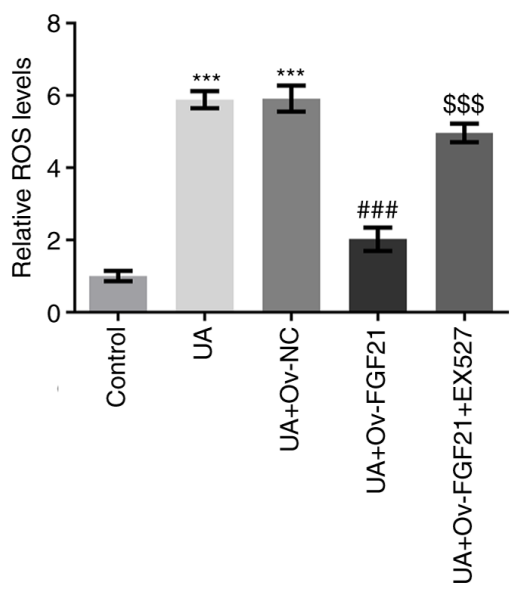

Figure 5. Overexpression of FGF21 attenuates oxidative stress in high UA-induced HUVECs by activating sirtuin 1. (A) ROS production was assessed via dichloro-dihydro-fluorescein diacetate staining (magnification, $\mathrm{x} 200$ ). (B) ROS levels were quantified using a ROS assay kit. ${ }^{* * *} \mathrm{P}<0.001$ vs. control; ${ }^{\# \# "} \mathrm{P}<0.001$ vs. UA + Ov-NC; ${ }^{\$ \$ \$} \mathrm{P}<0.001$ vs. UA + Ov-FGF21. FGF21, fibroblast growth factor 21; UA, uric acid; ROS, reactive oxygen species; Ov, overexpressed; $\mathrm{NC}$, negative control.
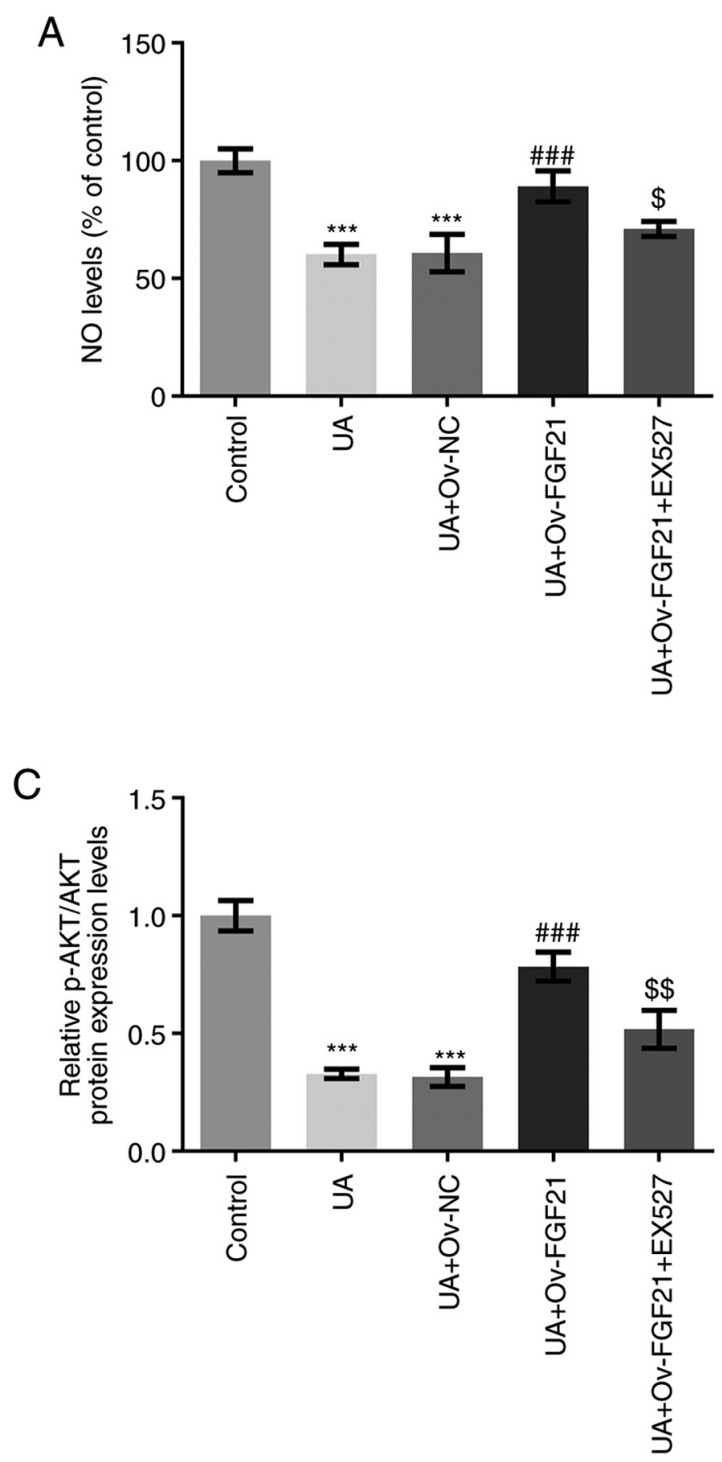

B
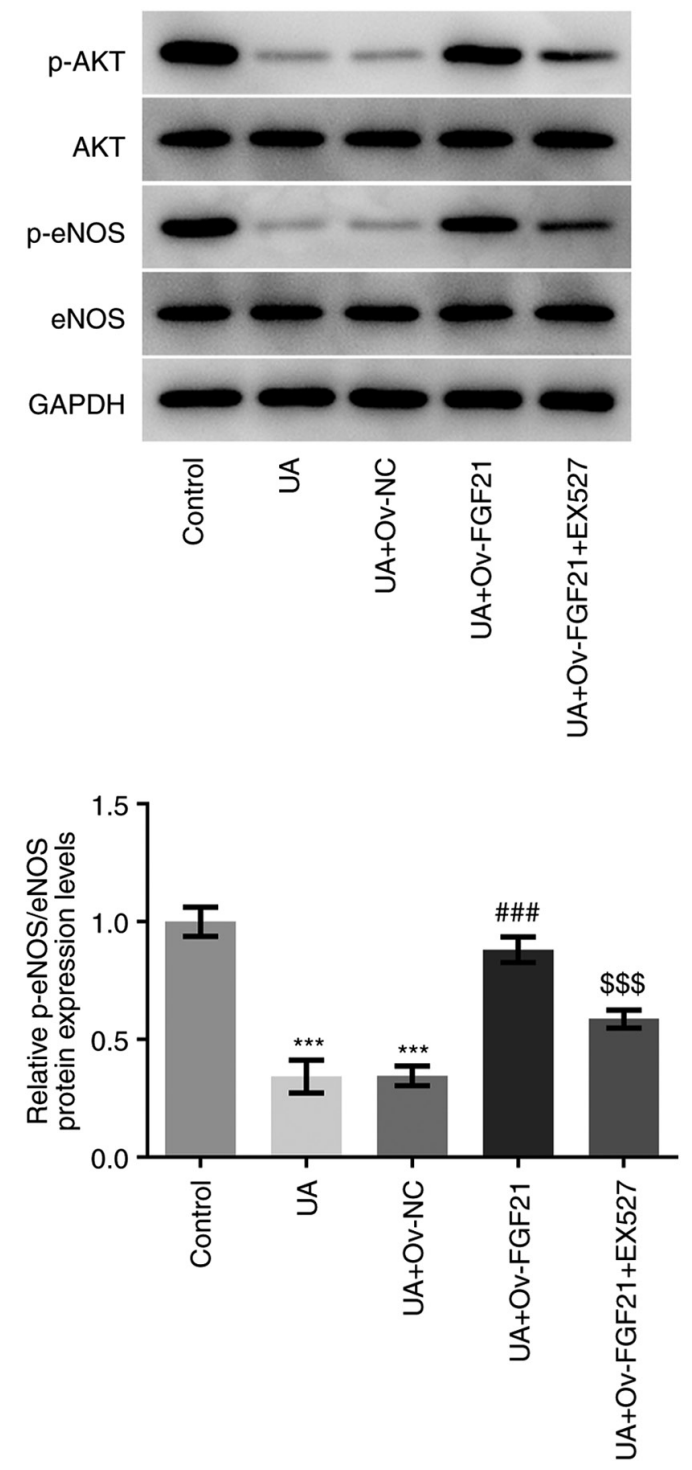

Figure 6. Overexpression of FGF21 attenuates vascular endothelial cell dysfunction in high UA-induced HUVECs by activating Sirtuin 1. (A) NO levels were quantified using a NO assay kit. (B) Western blotting was performed to detect the protein levels of p-AKT, AKT, p-eNOS and eNOS. (C) Semi-quantification of p-AKT/AKT and p-eNOS/eNOS ratios. ${ }^{* * *} \mathrm{P}<0.001$ vs. control; ${ }^{\# \# "} \mathrm{P}<0.001$ vs. $\mathrm{UA}+\mathrm{Ov}-\mathrm{NC} ;{ }^{\$} \mathrm{P}<0.05,{ }^{\$ \$} \mathrm{P}<0.01$ and ${ }^{\$ \$ \$} \mathrm{P}<0.001 \mathrm{vs}$. UA + Ov-FGF21. FGF21, fibroblast growth factor 21; UA, uric acid; NO, nitric oxide; eNOS, endothelial NO synthase; Ov, overexpressed; NC, negative control; p-, phosphorylated. 
ratios of $\mathrm{p}-\mathrm{AKT} / \mathrm{AKT}$ and $\mathrm{p}-\mathrm{eNOS} / \mathrm{eNOS}$ compared with those in the control group. These effects were significantly reversed following overexpression of FGF21 compared with those in the UA + Ov-NC group, which were partially but significantly reversed by the Sirt1 inhibitor EX527 compared with those in the $\mathrm{UA}+$ Ov-FGF21 group (Fig. 6B and C). These results suggest that EX527 may reverse the suppressive effects of FGF21 overexpression on vascular endothelial cell dysfunction. Therefore, overexpression of FGF21 may attenuate oxidative stress and vascular endothelial cell dysfunction in high UA-induced HUVECs by activating Sirt1.

\section{Discussion}

Hyperuricemia occurs when UA levels are elevated in the blood, increasing the risk of gout and nephrolithiasis (20). Hyperuricemia is considered a risk factor for a number of disorders, including diabetes mellitus, cardiovascular diseases and metabolic syndrome (21). Accumulating evidence suggests that UA can cause endothelial injury and dysfunction, though the mechanism remain unclear $(22,23)$. Apoptosis induced by ox-LDL in vascular endothelial cells is an important process in the progression of atherosclerosis (11). FGF21 has been reported to be closely associated with metabolic dysfunction, including the development of vascular calcification and atherosclerotic disease (24-26). In the present study, UA stimulation was found to significantly decrease FGF21 mRNA and protein expression, suggesting a potential association between FGF21 and UA-induced vascular endothelial cell injury. Therefore, the present study aimed to investigate the role of FGF21 in a vascular endothelial cell injury model induced by UA.

Activation of ER stress, which frequently occurs using diabetes and metabolic syndrome, can cause endothelial dysfunction (27). The eIF2A/ATF4/CHOP signaling pathway serves an important role in regulating ER stress (28). The results of the present study demonstrated that UA stimulation significantly activated ER stress. Notably, overexpression of FGF21 suppressed the activation of ER stress in UA-stimulated HUVECs, which suggested that FGF21 may exert protective effects on HUVECs against ER stress. To investigate the detailed mechanism underlying the role of FGF21 in UA-stimulated cells, a series of in vitro experiments were performed.

A previous study reported that FGF21 can protect against angiotensin II-induced cardiac hypertrophy and dysfunction in a Sirt1-dependent manner (29). Furthermore, FGF21 has been found to promote the formation of aerobic myofibers through the Sirtl-mediated signaling pathway (30). Results of the present study demonstrated that Sirt1 mRNA and protein expression were significantly decreased following UA stimulation. However, overexpression of FGF21 significantly increased Sirt1 expression. Therefore, the Sirt1 inhibitor, EX527, was used to determine whether Sirtl was a downstream molecule of FGF21. The results of the present study demonstrated that EX527 significantly reversed the suppressive effects of FGF21 overexpression on ER stress in UA-stimulated HUVECs. ER stress can activate the NLRP3 inflammasome to induce inflammatory responses and oxidative stress (31). However, FGF21 can ameliorate atherosclerosis by inhibiting NLRP3 inflammasome-related cell pyroptosis and ER stress in vascular endothelial cells (32). In the present study, overexpression of FGF21 significantly decreased the expression levels of inflammatory cytokines and components of the NLRP3 inflammasome, which were partially reversed by the Sirt1 inhibitor. Taken together, these results suggested that FGF21 may exert a suppressive effect on ER stress and inflammation by activating Sirt1.

Generation of ROS, reduction in eNOS activity and therefore decreased NO release are considered to be a prominent mechanism underlying endothelial injury (22). It has been previously reported that NO production is reduced in a the high UA-induced hyperuricemia model (22), which is consistent with the results of the present study. The results demonstrated that UA stimulation induced oxidative stress and vascular endothelial cell dysfunction, as demonstrated by the significantly increased ROS production, significant decline in NO release and the significant decrease in eNOS activity in UA-stimulated HUVECs in the present study. However, these effects were significantly reversed following overexpression of FGF21. EX527 significantly abrogated the suppressive effects of FGF21 overexpression on oxidative stress and vascular endothelial cell dysfunction. These results suggest that FGF21 may attenuate UA-induced ER stress, inflammation and vascular endothelial cell dysfunction by activating sirt1.

In conclusion, results of the present study suggest that FGF21 can attenuate ER stress, inflammatory injury and endothelial cell dysfunction caused by high UA by Sirt1 activation. However, the experimental sirt1 inhibitor, EX527, only partially reversed the effects of FGF21, suggesting that FGF21 may also act through other signaling pathways, which warrants further exploratory studies.

\section{Acknowledgements}

Not applicable.

\section{Funding}

The present study was supported by the Scientific Research Fund of Sichuan Health and Health Committee (grant no. 19PJ114).

\section{Availability of data and materials}

The datasets used and/or analyzed during the current study are available from the corresponding author on reasonable request.

\section{Authors' contributions}

RO, XZ, RZ and DD conceived and designed the study. RO, XZ, RZ, JY and SL collected and analyzed the data. All authors were involved in the writing of the manuscript and revised the manuscript. RO and DD confirm the authenticity of all the raw data. All authors read and approved the final version of the manuscript.

\section{Ethics approval and consent to participate}

Not applicable. 


\section{Patient consent for publication}

Not applicable.

\section{Competing interests}

The authors declare that they have no competing interests.

\section{References}

1. Yang X, Gu J, Lv H, Li H, Cheng Y, Liu Y and Jiang Y: Uric acid induced inflammatory responses in endothelial cells via up-regulating(pro)renin receptor. Biomed Pharmacother 109: 1163-1170, 2019

2. Ramirez-Sandoval JC and Madero M: Treatment of hyperuricemia in chronic kidney disease. Contrib Nephrol 192: 135-146, 2018.

3. Ng G, Chau EM and Shi Y: Recent developments in immune activation by uric acid crystals. Arch Immunol Ther Exp (Warsz) 58 273-277, 2010

4. Stodle GS, Silva GB, Tangeras LH, Gierman LM, Nervik I, Dahlberg UE, Sun C, Aune MH, Thomsen LCV, Bjørge L and Iversen AC: Placental inflammation in pre-eclampsia by Nod-like receptor protein (NLRP) 3 inflammasome activation in trophoblasts. Clin Exp Immunol 193: 84-94, 2018.

5. Zhang S, Wang Y, Cheng J, Huangfu N, Zhao R, Xu Z, Zhang F, Zheng $\mathrm{W}$ and Zhang D: Hyperuricemia and cardiovascular disease. Curr Pharm Des 25: 700-709, 2019.

6. Johnson RJ, Bakris GL, Borghi C, Chonchol MB, Feldman D, Lanaspa MA, Merriman TR, Moe OW, Mount DB, Sanchez Lozada LG, et al: Hyperuricemia, acute and chronic kidney disease, hypertension, and cardiovascular disease: Report of a scientific workshop organized by the national kidney foundation. Am J Kidney Dis 71: 851-865, 2018

7. Choi YJ, Yoon Y, Lee KY, Hien TT, Kang KW, Kim KC, Lee J, Lee MY, Lee SM, Kang DH and Lee BH: Uric acid induces endothelial dysfunction by vascular insulin resistance associated with the impairment of nitric oxide synthesis. FASEB J 28 : 3197-3204, 2014.

8. Assadi F: Allopurinol enhances the blood pressure lowering effect of enalapril in children with hyperuricemic essential hypertension. J Nephrol 27: 51-56, 2014.

9. Alonge KM, Meares GP and Hillgartner FB: Glucagon and insulin cooperatively stimulate fibroblast growth factor 21 gene transcription by increasing the expression of activating transcription factor 4. J Biol Chem 292: 5239-5252, 2017.

10. Brahma MK, Adam RC, Pollak NM, Jaeger D, Zierler KA Pöcher N, Schreiber R, Romauch M, Moustafa T, Eder S, et al: Fibroblast growth factor 21 is induced upon cardiac stress and alters cardiac lipid homeostasis. J Lipid Res 55: 2229-2241, 2014.

11. Yan X, Gou Z, Li Y, Wang Y, Zhu J, Xu G and Zhang Q Fibroblast growth factor 21 inhibits atherosclerosis in apoE-/mice by ameliorating Fas-mediated apoptosis. Lipids Health Dis 17: 203, 2018.

12. Chen JJ, Tao J, Zhang XL, Xia LZ, Zeng JF, Zhang H, Wei DH, Lv YC, Li GH and Wang Z: Inhibition of the ox-LDL-induced pyroptosis by FGF21 of human umbilical vein endothelial cells through the TET2-UQCRC1-ROS pathway. DNA Cell Biol 39: $661-670,2020$

13. Guo D, Xiao L, Hu H, Liu M, Yang L and Lin X: FGF21 protects human umbilical vein endothelial cells against high glucose-induced apoptosis via PI3K/Akt/Fox3a signaling pathway. J Diabetes Complications 32: 729-736, 2018.

14. Chen S, Chen D, Yang H, Wang X, Wang J and Xu C: Uric acid induced hepatocytes lipid accumulation through regulation of miR-149-5p/FGF21 axis. BMC Gastroenterol 20: 39, 2020.

15. Liu ZH, Zhang Y, Wang X, Fan XF, Zhang Y, Li X, Gong YS and Han LP: SIRT1 activation attenuates cardiac fibrosis by endothelial-to-mesenchymal transition. Biomed Pharmacother 118 109227, 2019.

16. Livak KJ and Schmittgen TD: Analysis of relative gene expression data using real-time quantitative PCR and the 2(-Delta Delta C(T)) method. Methods 25: 402-408, 2001.
17. Wu X, Pan C, Chen R, Zhang S, Zhai Y and Guo H: BML-111 attenuates high glucose-induced inflammation, oxidative stress and reduces extracellular matrix accumulation via targeting Nrf2 in rat glomerular mesangial cells. Int Immunopharmacol 79: $106108,2020$.

18. Aranda A, Sequedo L, Tolosa L, Quintas G, Burello E, Castell JV and Gombau L: Dichloro-dihydro-fluorescein diacetate (DCFH-DA) assay: A quantitative method for oxidative stress assessment of nanoparticle-treated cells. Toxicol In Vitro 27: 954-963, 2013.

19. Liu HD, Li W, Chen ZR, Hu YC, Zhang DD, Shen W, Zhou ML, Zhu L and Hang CH: Expression of the NLRP3 inflammasome in cerebral cortex after traumatic brain injury in a rat model. Neurochem Res 38: 2072-2083, 2013.

20. Li L, Zhang Y and Zeng C: Update on the epidemiology, genetics, and therapeutic options of hyperuricemia. Am J Transl Res 12: 3167-3181, 2020.

21. George C and Minter DA: Hyperuricemia. In: StatPearls (Internet). StatPearls Publishing, Treasure Island, FL, 2021.

22. Hong Q, Wang L, Huang Z, Feng Z, Cui S, Fu B, Cai G, Chen $X$ and Wu D: High concentrations of uric acid and angiotensin II act additively to produce endothelial injury. Mediators Inflamm 2020: 8387654, 2020

23. Yang B, Li S, Zhu J, Huang S, Zhang A, Jia Z, Ding G and Zhang Y: miR-214 protects against uric acid-induced endothelial cell apoptosis. Front Med (Lausanne) 7: 411, 2020.

24. Durnwald C, Mele L, Landon MB, Varner MW, Casey BM, Reddy UM, Wapner RJ, Rouse DJ, Tita ATN, Thorp JM Jr, et al: Fibroblast growth factor 21 and metabolic dysfunction in women with a prior glucose-intolerant pregnancy. Am J Perinatol 38: 1380-1385, 2020.

25. Lee SY, Burns SF, Ng KKC, Stensel DJ, Zhong L, Tan FHY, Chia KL, Fam KD, Yap MMC, Yeo KP, et al: Fibroblast growth factor 21 mediates the associations between exercise, aging, and glucose regulation. Med Sci Sports Exerc 52: 370-380, 2020.

26. Wu L, Qian L, Zhang L, Zhang J, Zhou J, Li Y, Hou X, Fang Q, $\mathrm{Li} \mathrm{H}$ and Jia W: Fibroblast growth factor 21 is related to atherosclerosis independent of nonalcoholic fatty liver disease and predicts atherosclerotic cardiovascular events. J Am Heart Assoc 9: e015226, 2020.

27. Osman A, El-Gamal H, Pasha M, Zeidan A, Korashy HM, Abdelsalam SS, Hasan M, Benameur T and Agouni A: Endoplasmic reticulum (ER) stress-generated extracellular vesicles (microparticles) self-perpetuate ER stress and mediate endothelial cell dysfunction independently of cell survival. Front Cardiovasc Med 7: 584791, 2020.

28. Guo Y, Guo R, Su Y, Fu J, Wang S, Kong Y, Wu C, Wang J, Tan C, Mo C and Zhao B: The PERK/eIF2alpha/ATF4/CHOP pathway plays a role in regulating monocrotaline-induced endoplasmic reticulum stress in rat liver. Res Vet Sci 130: 237-239, 2020.

29. Li S, Zhu Z, Xue M, Yi X, Liang J, Niu C, Chen G, Shen Y, Zhang $\mathrm{H}$, Zheng J, et al: Fibroblast growth factor 21 protects the heart from angiotensin II-induced cardiac hypertrophy and dysfunction via SIRT1. Biochim Biophys Acta Mol Basis Dis 1865: 1241-1252, 2019.

30. Liu X, Wang Y, Hou L, Xiong Y and Zhao S: Fibroblast growth factor 21 (FGF21) promotes formation of aerobic myofibers via the FGF21-SIRT1-AMPK-PGC1 $\alpha$ pathway. J Cell Physiol 232: 1893-1906, 2017

31. Li W, Cao T, Luo C, Cai J, Zhou X, Xiao X and Liu S: Crosstalk between ER stress, NLRP3 inflammasome, and inflammation. Appl Microbiol Biotechnol 104: 6129-6140, 2020.

32. Zeng Z, Zheng Q, Chen J, Tan X, Li Q, Ding L, Zhang R and Lin X: FGF21 mitigates atherosclerosis via inhibition of NLRP3 inflammasome-mediated vascular endothelial cells pyroptosis. Exp Cell Res 393: 112108, 2020.

This work is licensed under a Creative Commons Attribution-NonCommercial-NoDerivatives 4.0 International (CC BY-NC-ND 4.0) License. 